\title{
Characteristics and predictors of progression in an Egyptian multiple sclerosis cohort: a multicenter registry study
}

This article was published in the following Dove Press journal:

Neuropsychiatric Disease and Treatment

18 July 2017

Number of times this article has been viewed

\author{
Sherif M Hamdy' \\ Maged Abdel-Naseer ${ }^{\prime}$ \\ Nevin M Shalaby' \\ Alaa N Elmazny' \\ Ahmed A Nemr ${ }^{2}$ \\ Amr Hassan' \\ Mohamed I Hegazy' \\ Husam S Mourad' \\ Nirmeen A Kishk' \\ Mona A Nada' \\ Ahmed Abdelalim' \\ Amr M Fouad' \\ Hatem S Shehata' \\ 'Neurology Department, Cairo \\ University, ${ }^{2}$ Neurology Department, \\ Maadi Military Hospital, Cairo, Egypt
}

Correspondence: Nevin M Shalaby

Neurology Department, Cairo University,

Kasr Alainy School of Medicine -

Department I5, I Al-Saraya Street,

Al-Manial, Cairo II 432, Egypt

Tel +2010 01493242

Email nevinmohy@gmail.com
Background: Multiple sclerosis (MS) is a complex autoimmune disease with a heterogeneous presentation and diverse disease course. Recent studies indicate a rising prevalence of MS in the Middle East.

Objective: To characterize the demographics and disease features of Egyptian patients attending four tertiary referral MS centers in Cairo.

Materials and methods: This was a retrospective, observational study on 1,581 patients between 2001 and 2015. Medical records were reviewed and data were identified and extracted in a standardized electronic registry.

Results: The mean age of disease onset was $26.6 \pm 7.8$ years, with the majority being female (2.11:1). Relapsing-remitting MS was the most common type (75.1\%). The main presenting symptom was motor weakness (43.9\%), which was also the most frequent symptom during the disease course. Family history of MS was found in 2.28\%. Higher initial Expanded Disability Status Scale score, black holes, and infratentorial lesions on initial magnetic resonance imaging were independent factors for disease progression by univariate analysis (OR 3.87 [95\% CI 1.84-6.51], 4.14 [95\% CI 3.08-5.58], 4.07 [95\% CI 3.21-4.99], respectively); however, in multivariate analysis, only infratentorial lesions were an independent risk for disease progression (OR 6, 95\% CI 2.99-12.02; $P=0.0005$ ).

Conclusion: The results from this registry - the largest for MS in the Arab region to date - are comparable to other registries with slight differences.

Keywords: multiple sclerosis, MS registry, epidemiology, Middle East, North Africa, Egypt

\section{Introduction}

Multiple sclerosis (MS) is a chronic inflammatory central nervous system (CNS) disease, arising from a complex interaction of both environmental and genetic factors. ${ }^{1}$ MS affects individuals during the most productive time of their lives, and directly limits their work capacity, leading to major social and economic consequences. ${ }^{2}$

MS comprises manifestations of acute, as well as chronically accumulating symptoms, including numbness, weakness, optic neuritis, incoordination, diplopia, dizziness, and vertigo. The clinical profile of MS is largely different among various populations, based on both genetic and environmental factors. ${ }^{3}$ While Middle Eastern and North African (MENA) countries were previously considered areas of lowmoderate risk of $\mathrm{MS},{ }^{4}$ recent evidence now suggests a rising prevalence of MS in this region., 5

Despite being the most heavily populated country in the Arab world and the ME, limited data are currently available about the demographics and clinical characteristics 
of patients with MS in Egypt. ${ }^{7,8}$ This study aimed to depict the demographics and clinical and paraclinical features of patients attending four tertiary referral MS centers in Egypt.

\section{Materials and methods}

This study was registered with ClinicalTrials.gov (NCT03004794) on December 23, 2016. It was conducted in accordance with the principles established by the 18th World Medical Assembly (Helsinki, 1964) and all applicable amendments laid down by it, International Council for Harmonisation guidelines for good clinical practice, and in compliance with all national and international laws and regulations. Written informed consent was obtained from all participants in this study.

\section{Study design}

This was an observational, retrospective study on 1,581 patients attending four tertiary referral centers (2001-2015): Kasr Al-Ainy Multiple Sclerosis Research Unit (KAMSU; Cairo University Hospitals), Maadi Military Hospital, and two private centers in Cairo, Egypt. Every patient was registered using unique 14-digit identification number (mandatory in Egypt since 1999) to prevent duplicate entries.

\section{Patient record-review procedure and sampling strategies}

Medical records, administrative data, and laboratory and diagnostic tests reports were screened by three authors (HS, NS, and AE), during March-September 2015 after signed informed consent form had been obtained from all patients, and then relevant data were extracted to a standard electronic form according to the KAMSU registry. An assigned coordinator from each center facilitated data collection from site-specific records. In cases of incomplete documentation or unclear information, an on-site data coordinator performed data verification by phone or during face-to-face interviews with patients during routine follow-up or unscheduled visits. In cases of failure to complete missing documentation, the entire case was excluded from the analysis. Several measures were taken to ensure the confidentiality of the information collected. Patients' identification was kept confidential, and no names were included in the database presented by site coordinators. Moreover, the study staff signed a confidentiality agreement for nondisclosure. The study protocol was approved by the Neurology Department Review Board at Cairo University.

\section{Study population and diagnostic criteria}

The medical records of 1,581 patients were reviewed and the diagnosis of MS revised according to the 2010 revision of the McDonald criteria. ${ }^{9}$ Based on the disease course, patients were classified as relapsing-remitting MS (RRMS), secondary progressive MS (SPMS), and primary progressive MS (PPMS) ${ }^{10}$ Patients with a first demyelinating event prior to 2010 were reassessed by on-site coordinators and categorized with clinically isolated syndrome (CIS) whenever applicable, according to the 2010 McDonald criteria. Neuromyelitis optica (NMO) diagnosis was verified according to the 2015 diagnostic criteria. ${ }^{11}$ Those with typical brain magnetic resonance imaging (MRI) MSlike lesions without related clinical manifestations were diagnosed as radiologically isolated syndrome (RIS). ${ }^{12}$ Other patients included were those with unsettled diagnoses or MS mimics ( $n=57$ : Behçet's [ $n=11]$, vascular $[\mathrm{n}=9]$, acute disseminated encephalomyelitis [ $\mathrm{n}=9$ ], lupus [ $n=7]$, sarcoid $[n=6]$, other vasculitis $[n=5]$, CNS lymphoma [n=3], toxic $[n=3]$, tuberculosis $[n=2]$, and leukodystrophy [n=2]) (Figure 1).

Termination of the follow-up period was considered to be at the time of the last documented Expanded Disability Status Scale (EDSS) assessment. Relapse was defined according to the McDonald criteria, and the annualized relapse rate (ARR) was defined as the number of confirmed relapses per year. ${ }^{9}$

\section{Laboratory testing}

Serum and CSF samples were collected. CSF samples were analyzed for chemistry, cytology, IgG index, and oligoclonal bands using either gel electrophoresis or isoelectric focusing techniques. IgG antibodies were assessed in sera. Aqp4 antibodies by enzyme-linked immunosorbent assays were requested in suspected NMO-spectrum disorder (NMOSD) cases. Additional laboratory testing, including collagen vascular profiles, was done when necessary.

\section{Magnetic resonance imaging}

A total of 2,536 brain and 1,007 cervical spinal cord initial and follow-up MRI results were reevaluated. Since this was a retrospective study, a variety of imaging protocols and scanners were utilized. Imaging results were reviewed by on-site coordinators, and the extracted data were standardized for certain radiographic features, including typical vs atypical lesions, supratentorial vs infratentorial location, active lesions, and black holes.

\section{Electrophysiological testing}

Electrophysiological testing included visual evoked potential (VEP; $n=823$ ), auditory EP (AEP; $n=491$ ), and somatosensory EP (SSEP; $\mathrm{n}=307)$. 


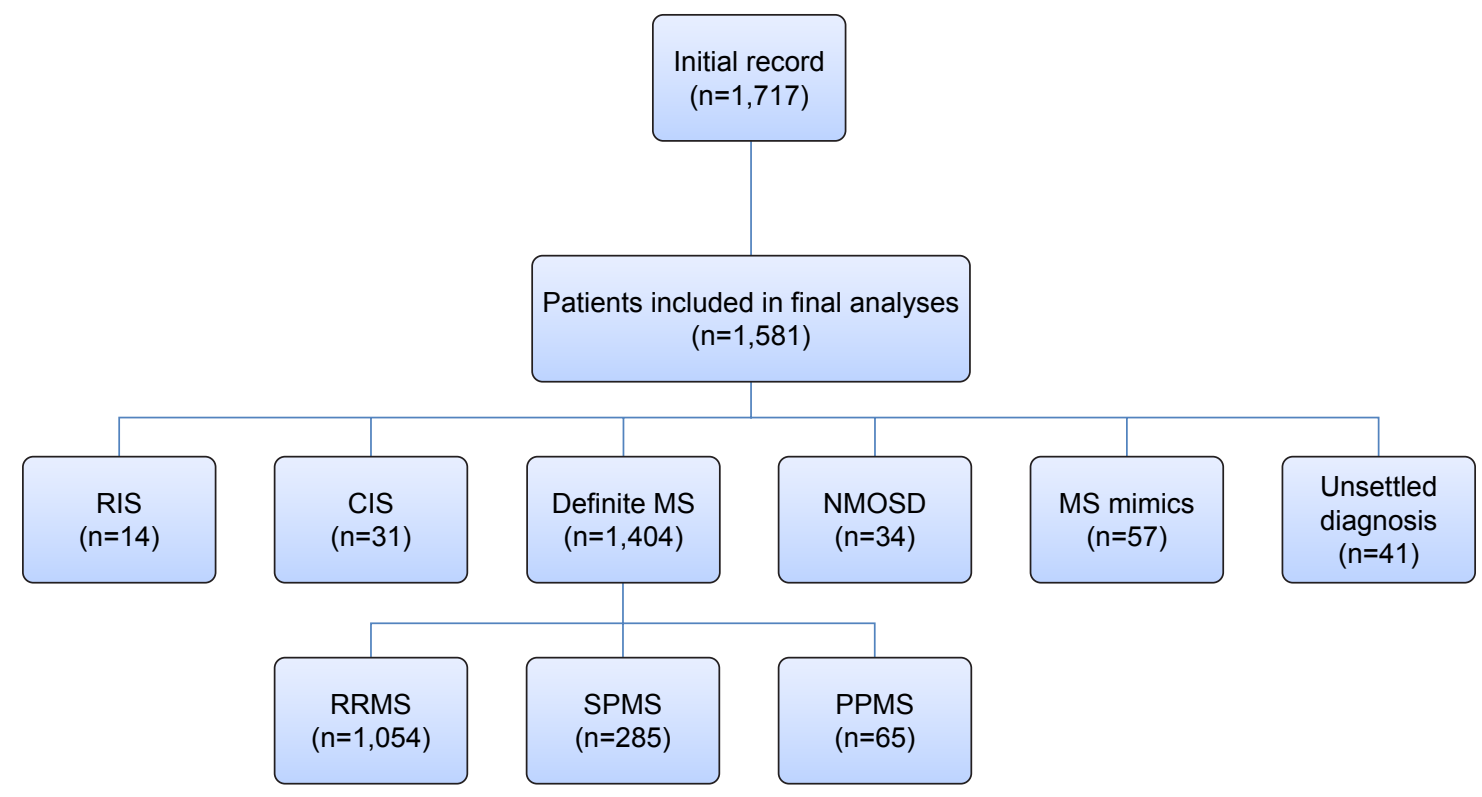

Figure I Disposition of the study population.

Abbreviations: RIS, radiologically isolated syndrome; CIS, clinically isolated syndrome; MS, multiple sclerosis; NMOSD, neuromyelitis optica-spectrum disorder; RRMS, relapsing-remitting MS; SPMS, secondary progressive MS; PPMS, primary progressive MS.

\section{Statistical analysis}

SPSS version 23.0 was used for data management and data analysis. Means \pm SD described quantitative variables, and medians were used for nonuniform values. Numbers and percentages described qualitative data and $\chi^{2}$ values nonparametric data. For comparison of mean values of two independent groups, Student's $t$-test was used. Multivariate logistic regression analysis was used to depict independent variables (demographic, clinical, and paraclinical data) that affected the likelihood (expressed as OR and 95\% CI) of developing SPMS, which was developed by sequentially adding variables with significant ORs in univariate analyses. $P$-values were always two-tailed and significant at the 0.05 level. The area under the receiver-operating characteristic (ROC) curve (AUC) was computed from each logistic regression result. The ROC curve plots the true-positive rate (predicted secondary progression that actually occurred) against the false-positive rate (predicted secondary progression that did not actually occur), and the AUC provides a summary measure of the accuracy of the potential predictive variables. The AUC ranges in value from 0.5 (chance) to 1 (perfect accuracy).

\section{Results}

\section{Demographic data and diagnostic scheme of all included patients}

Initially, medical records of 1,717 patients were reviewed, and $136(7.9 \%)$ were subsequently excluded due to missing data that could not be obtained. Of the 1,581 patients included, 1,078 (68.2\%) were females. Enrolled subjects had a wide distribution of ages: 6-60 years, with a mean age of $29.94 \pm 8.65$ years. Greater Cairo was the city of residence for the majority of patients $(1,139$ of 1,581, 72\%). About a third of our cohort $(n=514,32.5 \%)$ had left the workforce, and $44(2.8 \%)$ had changed jobs because of their illness. Divorce due to disease took place in 157 patients (9.9\%), about two-thirds of which were women $(n=105)$.

By the time of data collection, MS had been diagnosed in 1,404 patients (88.8\%); the diagnoses of 41 patients $(2.6 \%)$ had not yet been settled. Demographics by diagnostic category are summarized in Table 1. Concerning age at disease onset, pediatric onset of MS ( $<18$ years) was reported in 97 of 1,404 (6.9\%) patients, while $49(3.5 \%)$ had late-onset ( $>50$ years) MS.

\section{Clinical characteristics of MS}

The most common type was RRMS (1,054 of 1,404 [75.1\%]); SPMS was diagnosed in 285 patients (20.3\%) and PPMS in $65(4.6 \%)$. The main presenting symptoms were motor symptoms (616 of 1,404 [43.9\%]), followed by sensory symptoms (465 of 1,404 [33.1\%]), while 54 patients (3.9\%) had unusual presentations (Table 2). About a third of our cases had polysymptomatic disease onset $(n=471$ [33.6\%]). For all centers, the follow-up period ranged from 1 to 156 months, with a mean of $53.2 \pm 78.3$ months (median 39 months). During this period, 13 patients $(0.9 \%)$ died from 
Table I Demographics of included patients by diagnostic category

\begin{tabular}{|c|c|c|c|c|c|c|}
\hline & $\begin{array}{l}\text { Study population } \\
n=I, 58 I\end{array}$ & $\begin{array}{l}\text { MS } \\
n=I, 404(88.8 \%)\end{array}$ & $\begin{array}{l}\text { CIS } \\
n=3 \text { I (2\%) }\end{array}$ & $\begin{array}{l}\text { RIS } \\
n=I 4(0.9 \%)\end{array}$ & $\begin{array}{l}\text { NMOSD } \\
n=34(2.2 \%)\end{array}$ & $\begin{array}{l}\text { MS mimics } \\
n=57(3.6 \%)\end{array}$ \\
\hline \multicolumn{7}{|c|}{ Age at disease onset (years) } \\
\hline Range & $6-60$ & $6-58$ & $17.5-42$ & $16-49$ & $|5-5|$ & $11-60$ \\
\hline Mean \pm SD & $27.32 \pm 7.67$ & $26.61 \pm 7.82$ & $25.7 I \pm 6.94$ & $25.98 \pm 8.11$ & $27.31 \pm 8.82$ & $33.71 \pm 8.12$ \\
\hline Median & 29.22 & 26.59 & 25.25 & 25.53 & 31.25 & 33.58 \\
\hline \multicolumn{7}{|l|}{ Sex } \\
\hline Female/male & $\mathrm{I}, 078 / 503$ & $953 / 451$ & $23 / 8$ & $10 / 4$ & $25 / 9$ & $39 / 18$ \\
\hline Ratio & $2.14: 1$ & $2.11: I$ & 2.88:I & $2.50: 1$ & 2.78:I & $2.17: 1$ \\
\hline \multicolumn{7}{|c|}{ Disease duration (months) } \\
\hline Range & $\mathrm{I}-257$ & $\mathrm{I}-257$ & $1-62$ & $1-13$ & $\mathrm{I}-250$ & $\mathrm{I}-254$ \\
\hline Mean \pm SD & $83.69 \pm 91.79$ & $91.35 \pm 83.11$ & $5 I .31 \pm 28.1$ & $4.95 \pm 3.21$ & $61.39 \pm 76.84$ & $28.38 \pm 41.92$ \\
\hline Median & 72.92 & 78.04 & 249.99 & 5.27 & 63.71 & 37.19 \\
\hline \multicolumn{7}{|c|}{ Duration to diagnosis (months) } \\
\hline Range & $\mathrm{I}-252$ & $\mathrm{I}-252$ & $\mathrm{I}-4.5$ & $\mathrm{I}-3$ & $\mathrm{I}-249$ & $|-25|$ \\
\hline Mean \pm SD & $20.15 \pm 37.82$ & $19.93 \pm 36.24$ & $2.11 \pm 1.87$ & $1.47 \pm 0.91$ & $25.3 \pm 38.7$ & $23.2 \pm 36.4$ \\
\hline Median & 23.98 & 24.08 & 2.01 & 1.31 & 26.75 & 25.81 \\
\hline
\end{tabular}

Abbreviations: MS, multiple sclerosis; CIS, clinically isolated syndrome; RIS, radiologically isolated syndrome; NMOSD, neuromyelitis optica-spectrum disorder.

MS-related complications. A total of 32 patients $(2.28 \%)$ reported a family history of MS.

\section{Symptoms developing during the course of the disease}

Motor weakness was the most common symptom experienced, followed by sensory symptoms. On the other hand, seizures and sensorineural hearing loss were the least encountered symptoms. The frequency of symptoms

Table 2 Clinical data of patients with multiple sclerosis $(n=I, 404)$

\begin{tabular}{ll}
\hline $\begin{array}{l}\text { Annualized relapse rate, range } \\
\text { (mean } \pm \text { SD) }\end{array}$ & $0.5-4(0.86 \pm 0.69)$ \\
Presenting symptoms, $n(\%)$ & $616(43.87)$ \\
Motor & $465(33.12)$ \\
Superficial sensory & $392(27.92)$ \\
Visual (optic) & $302(21.5 \mathrm{I})$ \\
Ataxia & $122(8.69)$ \\
Diplopia (oculomotor) & \\
Unusual presentation ( $=54,3.85 \%)$ & $13(0.93)$ \\
$\quad$ Cognitive deficit & $12(0.85)$ \\
$\quad$ Seizure disorders & $8(0.57)$ \\
$\quad$ Behavioral & $6(0.43)$ \\
$\quad$ Sexual dysfunction & $6(0.43)$ \\
$\quad$ Depression & $5(0.36)$ \\
$\quad$ Dystonia & $2(0.14)$ \\
$\quad$ Disturbed consciousness & $2(0.14)$ \\
$\quad$ Excessive daytime sleepiness & $0.5-8.5,2.85 \pm \mathrm{I} .44,3$ \\
Initial EDSS, range, mean \pm SD, median & $\mathrm{I}-10,4.65 \pm \mathrm{I} .98,3.5$ \\
Last assessed EDSS, range, & \\
mean \pm SD, median & $\mathrm{I}-266.8,3 \mathrm{I} .7 \pm 42.1,26.0 \mathrm{I}$ \\
Duration to treatment (months), range, & \\
mean \pm SD, median & \\
Family history, $\mathrm{n}$ (\%) & $32(2.28)$ \\
Multiple sclerosis & $54(3.85)$ \\
Other autoimmune diseases & \\
\hline
\end{tabular}

Abbreviation: EDSS, Expanded Disability Status Scale. developed during the course of the disease in MS patients is illustrated in Figure 2.

\section{Radiological and paraclinical findings}

Initial brain MRI scans were conducted for all participants, while initial cervical spinal cord scans were done for 1,007 of 1,581 patients $(63.7 \%$; all patients with NMOSD [n=34], 863 MS patients [61.47\%], 23 CIS patients [74.19\%], and 87 for other diagnoses [59.59\%]). The number of MRI scans for each patient ranged from one to 13 scans (median four scans), while the median number of annual follow-up scans requested was two (zero to three). The median duration between the initial and follow-up brain MRIs was 22.3 months. CSF analyses were conducted for 334 of 1,581 (21.1\%) patients, and serum AQP4 antibodies were assessed in 77 of 1,581 patients $(4.9 \%$; 23 revealed positive results, all with NMOSD [ $n=34,67.6 \%]$ ). Results of MRI, CSF analysis, and EP studies are shown in Table 3.

\section{MS patients' therapeutic path}

Initial disease-modifying therapies/ immunosuppressants

Initially, 1,113 (79.3\%) patients received immunomodulatory therapies (primarily IFN $\beta ; 448$ of 1,404 [31.9\%]), whereas 265 patients (18.87\%) did not receive treatment. In the last assessment of therapeutic options, 224 patients (15.95\%) were on no treatment (Table 4).

Therapeutic strategy modification during the course of the disease

During the follow-up period, 243 of 1,113 patients (21.8\%) shifted to another therapeutic line. Of those, 61 patients 


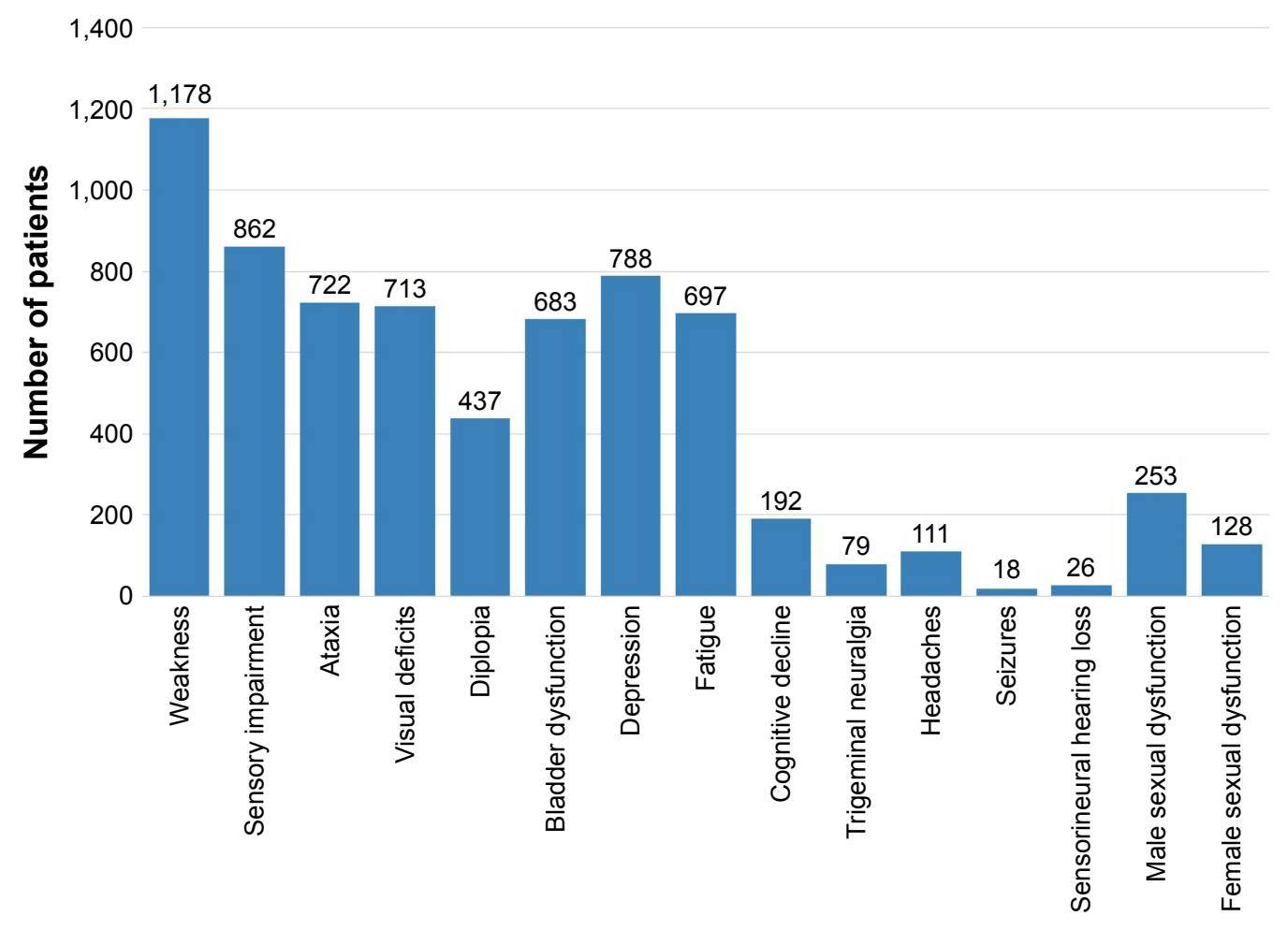

Figure 2 Symptom frequency during disease course in multiple sclerosis patients.

(25.1\%) had undergone further plan modification with subsequent changing of the therapeutic map repeatedly. Such modifications were due to recurrent relapses (41 of 243 [16.9\%]) or disease worsening (59 of 243 [24.3\%]) with subsequent escalation and/or induction therapies, and recent availability of disease-modifying therapies (DMTs; IFN $\beta$ and fingolimod) under the umbrella of the Ministry of Health (89 of 243 [36.6\%]). Temporary modifications for specific situations, such as pregnancy and/or lactation, were done for 54 patients (22.2\%). In the last assessment, 224 patients $(15.95 \%)$ were on no treatment (Table 4).

\section{Predictors of conversion to SPMS}

Univariate analysis included age at disease onset, ARR, disease duration, higher initial EDSS score, and initial brain MRI results (enhancing lesions, infratentorial lesions, and

Table 3 Radiological and paraclinical findings of MS, CIS, and NMOSD participants

\begin{tabular}{|c|c|c|c|c|}
\hline \multirow[b]{2}{*}{ Brain MRI } & \multicolumn{2}{|l|}{ MS cohort, $n=I, 404$} & \multirow{2}{*}{$\frac{\text { CIS, } n=3 \text { I }}{\text { Last reviewed MRI }}$} & \multirow{2}{*}{$\frac{\text { NMOSD, } n=34}{\text { Last reviewed MRI }}$} \\
\hline & Initial MRI $(n=I, 404)$ & Last follow-up MRI (n=I, I87) & & \\
\hline Typical lesions for MS & $I, 36 I$ (96.93) & I,I65/I,I87 (98.I5) & II (35.48) & 0 \\
\hline Atypical lesions for MS & $12(0.86)$ & $9 / 1,187(0.76)$ & 15 (48.39) & $23(67.65)$ \\
\hline Normal MRI & $3 I(2.2 I)$ & |3/I, I87 (1.09) & $5(16.13)$ & II (32.35) \\
\hline Active lesions $\left(\mathrm{Gd}^{+}\right)$ & $296(21.08)$ & $3|7 / I|$,87 (26.7I) & 0 & $5(|4.7|)$ \\
\hline Black holes & $128(9.12)$ & $331 / 1,187$ (27.89) & 0 & $7(20.59)$ \\
\hline Infratentorial lesions & $44 I(3 I .4 I)$ & $50 I / I, I 87(42.2 I)$ & $3 / 31(9.68)$ & $6(17.65)$ \\
\hline Initial cervical MRI (n) & $863 / 1,404$ & & $23 / 31$ & $34 / 34$ \\
\hline Positive & $647 / 863(74.97)$ & & $8 / 23(34.78)$ & $34(100)$ \\
\hline Normal & $216 / 863(25.03)$ & & $15 / 23(65.22)$ & 0 \\
\hline CSF analysis (n) & $227 / 1,404$ & & $|2 / 3|$ & $26 / 34$ \\
\hline Elevated CSF IgG index & I6I/227 (70.93) & & $3 / 12(25)$ & $1 / 26(3.85)$ \\
\hline Positive OCBs & I 54/227 (67.84) & & $4 / 12(33.33)$ & $\mathrm{I} / 26(3.85)$ \\
\hline \multicolumn{5}{|l|}{ Evoked responses } \\
\hline Abnormal VEP & $503 / 696(72.27)$ & & $15 / 22(68.18)$ & I5/2I (7I.43) \\
\hline Abnormal AEP & $262 / 4 \mid 4(63.29)$ & & $10 / 18(55.56)$ & I I/I7 (64.7I) \\
\hline Abnormal SSEP & 167/249 (67.07) & & $9 / 17$ (52.94) & $8 / 13(6 \mid .54)$ \\
\hline
\end{tabular}

Abbreviations: MS, multiple sclerosis; CIS, clinically isolated syndrome; NMOSD, neuromyelitis optica-spectrum disorder; MRI, magnetic resonance imaging; $\mathrm{Gd}^{+}$, Gadolinium enhancement; CSF, cerebrospinal fluid; OCBs, oligoclonal bands;VEP, visual evoked potential;AEP, auditory EP; SSEP, somatosensory EP. 
Table 4 Initial- and last-assessment treatment options for MS patients $(n=I, 404)$

\begin{tabular}{|c|c|c|}
\hline & $\begin{array}{l}\text { Initial } \\
\text { therapies }\end{array}$ & $\begin{array}{l}\text { Therapies at } \\
\text { final assessment }\end{array}$ \\
\hline $\begin{array}{l}\text { Disease-modifying } \\
\text { therapies, total, } \mathrm{n}(\%)\end{array}$ & $713(50.7)$ & $849(60.5)$ \\
\hline IFN $\beta$ & $448(31.91)$ & $603(42.95)$ \\
\hline Glatiramer acetate & $9(0.64)$ & II (0.78) \\
\hline Fingolimod & $19(1.35)$ & $8 \mathrm{I}(5.77)$ \\
\hline Leflunomide & $15(1.07)$ & $12(0.85)$ \\
\hline Natalizumab & $13(0.93)$ & $24(1.7 I)$ \\
\hline Rituximab & $15(1.07)$ & $17(1.21)$ \\
\hline Dimethyl fumarate & $8(0.57)$ & $6(0.43)$ \\
\hline Teriflunomide & $3(0.2 I)$ & $6(0.43)$ \\
\hline Monthly IVMP & $183(13.03)$ & $89(6.34)$ \\
\hline $\begin{array}{l}\text { Immunosuppressants, } \\
\text { total, n (\%) }\end{array}$ & $426(30.34)$ & $331(23.58)$ \\
\hline Cyclophosphamide & $140(9.97)$ & $145(10.33)$ \\
\hline Azathioprine & $164(11.68)$ & $91(6.48)$ \\
\hline Methotrexate & $43(3.06)$ & $54(3.85)$ \\
\hline Mitoxantrone & $79(5.63)$ & $4 \mathrm{I}(2.92)$ \\
\hline Total number treated & I, II $3(79.3)$ & $\mathrm{I}, 180(84)$ \\
\hline No treatment, total, n (\%) & $265(18.87)$ & $224(15.95)$ \\
\hline RRMS $(n=I, 054)$ & $123 / I, 054(\mid 1.67)$ & $73(6.92)$ \\
\hline SPMS $(n=285)$ & 109/285 (38.25) & $117(41.05)$ \\
\hline PPMS $(n=65)$ & $33 / 65(50.77)$ & $34(52.31)$ \\
\hline
\end{tabular}

Note: A total of 26 patients were initially on combination therapy.

Abbreviations: IVMP, intravenous methylprednisolone; MS, multiple sclerosis; RRMS, relapsing-remitting MS; SPMS, secondary progressive MS; PPMS, primary progressive MS.

black holes) as independent variables and MS type (RRMS vs SPMS) as the dichotomous target variable. A significant predictive value by was detected with higher initial EDSS score (OR 3.87, 95\% CI 1.84-6.51), presence of black holes (OR 4.14, 95\% CI 3.08-5.58), and infratentorial lesions (OR 4.07, 95\% CI 3.21-4.99) in initial brain MRI scans. Multivariate ROC-curve analyses revealed infratentorial lesions as the sole independent risk factor for SPMS development (AUC 0.682, OR 6, 95\% CI 2.99-12.02; $P=0.0005$ ) (Figure 3).

\section{Discussion}

In our study, the mean age at MS onset was $26.61 \pm 7.82$ years, slightly lower than the reported overall estimate in a meta-analysis of 52 studies in the MENA region (27.6129.48 years $)^{13}$ and much younger than that reported in the North American Research Committee on Multiple Sclerosis registry, where the age of onset was $31.4 \pm 9.7$ years $^{14}$ and in European MS registries, with MS symptoms starting around the mid-30s. ${ }^{15}$ For NMOSD, the mean age at disease onset was $27.31 \pm 8.82$ years, which is consistent with a Chinese study that showed no significant differences in mean age at
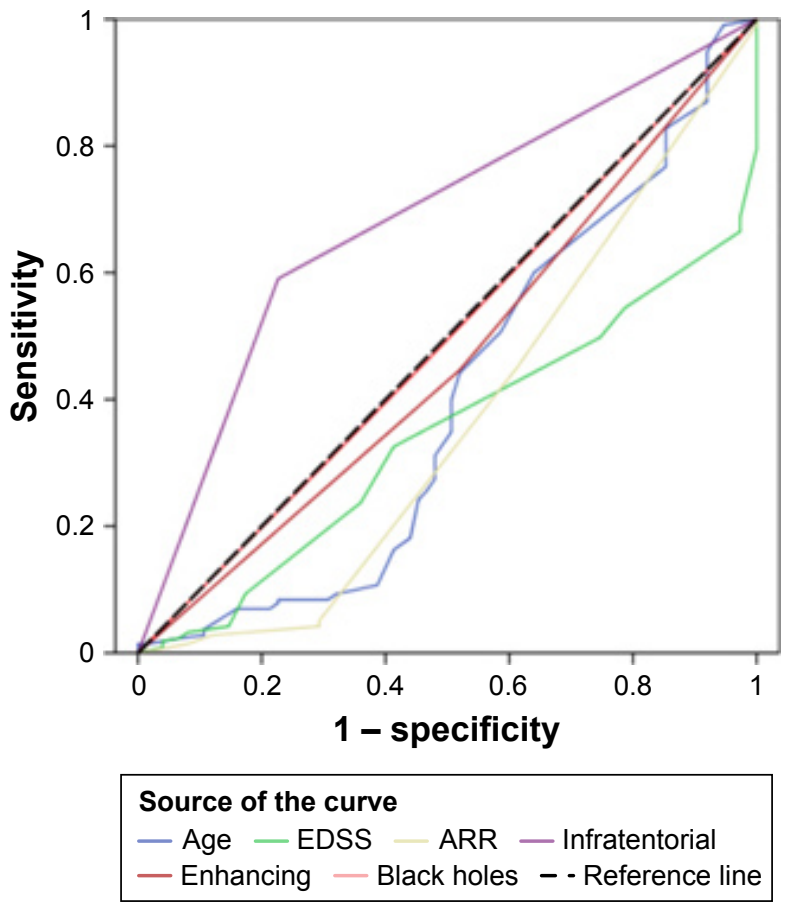

Figure 3 ROC curve analysis.

Note: Diagonal segments are producted by ties.

Abbreviations: ROC, receiver-operating characteristic; EDSS, Expanded Disability Status Scale; ARR, annualized relapse rate.

disease onset between MS and NMOSD patients. ${ }^{16}$ However, a typical distinguishing feature of NMOSD is the later age of onset - NMOSD patients are on average 10 years older than those with MS at disease presentation ${ }^{17}$ - which was not the case in our cohort.

Our results also confirmed female dominance in both MS and NMOSD, with female:male ratios of 2.14:1, and 2.78:1, respectively. While different studies have report a variety of female:male incidence ratios, both diseases are more prominent in females. Furthermore, this disparity in incidence between men and women is more apparent in NMOSD (ratio 1.9-7.3:1) compared to MS (ratio 2.57-2.62:1). ${ }^{18}$ According to previous reports, the female:male ratio varies widely in the Arab world, eg, from 0.8 in Oman to 4.3 in Saudi Arabia, and the overall estimate of female proportion in MS patients was $0.67(0.65-0.69){ }^{13}$

In the current series, $32.5 \%$ had quit their jobs and $2.8 \%$ modified their work due to the disease. Therefore, MS represents a major economic burden for patients and the community at large. Furthermore, high divorce rates (9.9\%) were observed in this study, indicating that MS also results in a tremendous social impact on patients. A study in England showed that the marital status of most patients had not changed since the onset of MS, but 53\% had given up 
their jobs as a direct result of the disease. ${ }^{19}$ The discrepancy in divorce rates can be attributed to the difference in cultural and religious factors between the two countries, as in Egypt the divorce process is easier than in Europe generally.

Family history of MS was reported in $2.3 \%$ of MS patients. In a Danish cohort, the relative lifetime risk of MS increased sevenfold among first-degree relatives, and the familial lifetime risk for first-degree relatives was $2.5 \%{ }^{20}$ AlemanyRodríguez et al found $5.9 \%$ of MS patients having a first- and/ or second-degree relative with MS. ${ }^{21}$ In Arab populations, higher family aggregation of MS was reported in Lebanon (5\%), Jordan (9.4\%), and Qatar (10.4\%). ${ }^{13}$ This difference is likely attributable to diversity in study designs, degree of case ascertainment, and cultural barriers (families may avoid sharing the precise history of chronic or debilitating diseases).

The median duration from symptom onset to diagnosis in our series was 24.1 months, which is similar to the Novo study, where the median time to diagnosis was 24.9 months. ${ }^{22}$ This is explained by the lack of awareness of MS symptoms, especially symptoms like numbness and diplopia, slight visual blurring that can resolve spontaneously and pass unnoticed.

The most common type of MS in our study was RRMS (75\%), followed by SPMS (20\%), then PPMS (5\%). Most literature reported RRMS in $85 \%$ of cases at onset. ${ }^{23,24}$ Other studies reported lower percentage of RRMS (66\%) and higher percentage of PPMS $(15 \%){ }^{25}$ A meta-analysis reported a pooled estimate in MENA for RRMS, SPMS, and PPMS to be $71 \%, 12 \%$, and $8 \%$ respectively. ${ }^{13}$

In our series, motor symptoms were the initial presentation in $43.9 \%$, followed by sensory and visual manifestations and ataxia (33.1\%, $27.9 \%$, and $21.5 \%$, respectively). Our findings are in agreement with several studies previously conducted in the MENA region. ${ }^{13,23,25}$ However, higher percentages for initial sensory symptoms and $d^{5}$ brain stem-cerebellar ${ }^{24}$ and optic neuritis were also reported. ${ }^{13}$ Unusual presenting symptoms were reported in $3.9 \%$ of our MS patients, more than a third of which were cognitive deficits and behavioral problems $(1.5 \%)$, followed by seizures $(0.9 \%)$. Kolber et al emphasized the role of gray-matter pathology and cortical lesions in early MS, which are believed to precede the appearance of the classic white-matter lesions and systematically correlate with patients' cognitive complaints and seizures. ${ }^{26}$ Moreover, the incidence of seizures among MS patients was reportedly higher than that in the general population, and it could be an initial presentation of the disease. ${ }^{27}$

Concerning the frequency of symptoms developing during the course of the disease, motor weakness was the most commonly reported symptom, followed by sensory deficits with frequently encountered depression and fatigue. The frequency of symptoms developing during the course of the disease was again comparable to other studies. ${ }^{5,13,24}$

The proportion of CIS patients in our study ( $2 \%)$ was markedly lower compared to rates reported for Qatari patients (14\%) and in Dubai (United Arab Emirates). ${ }^{5,28}$ However, both those studies included a smaller number of patients (154 and 284, respectively). The follow-up period was not disclosed in the Qatari series and was only 7 years in the Dubai study. These differences may explain the contradiction in observed CIS rates.

Only $16.2 \%$ of our MS sample had CSF analyses performed. Over $30 \%$ of patients with MS were oligoclonal band-negative. This high negative percentage was due to the former use of gel electrophoresis in our institutes, which was just recently replaced by isoelectric focusing, raising the sensitivity from $50 \%$ to over $95 \% .{ }^{29}$ The value of CSF analyses for MS patients remains debatable. While the potential of CSF biomarkers to mirror CNS pathology is significant, the lack of specificity of abnormalities detected in CSF analyses to confirm the diagnosis of MS and the relatively invasive nature of this maneuver render performing it nonmandatory, especially in the context of the McDonald criteria. ${ }^{30}$

Regarding the determinants of disease progression in our cohort, univariate analyses revealed that higher initial EDSS score and the presence of black holes and infratentorial lesions in the initial brain MRI carry higher risks for developing SPMS. Though ARR, total number of relapses, disease duration, longer time to treatment, and active lesions were highly significant in SPMS, they had a limited impact on disability. In multivariate analysis, only infratentorial lesions were considered a risk for progression to SPMS. These findings coincide with Minneboo et al, who emphasized the role of infratentorial lesions in long-term prognosis for patients with initial findings suggestive of MS. ${ }^{31}$ Moreover, investigations in the past few decades have revealed that many clinical and MRI factors are predictive of long-term outcomes, including sex, higher age and pyramidal symptoms at disease onset, higher baseline $T_{2}$ lesion load, early brain atrophy, shorter time to second clinical attack, and number of relapses in the early disease phase. However, the heterogeneity of individual disease courses makes prognostic statements highly difficult, and a comprehensive MS-outcome prediction model combining multiple variables is still lacking. ${ }^{32,33}$

In our sample, $67.6 \%$ of NMOSD patients had seropositive Aqp4-specific antibodies. This could partly be 
attributed to assay sensitivity. We used cell-based assays for a fraction of our cohort. This method has been shown to be both more sensitive and more specific than the previous gold standard: enzyme-linked immunosorbent assays and some immunofluorescence assays. ${ }^{34}$ However, Jarius and Wildemann reported that despite the use of sensitive assays, Aqp4-specific antibodies are not detected in up to $10 \%-40 \%$ of patients diagnosed with NMOSD. ${ }^{35}$

In the last assessment, $84 \%$ of our patients were receiving immunomodulatory treatment, while $16 \%$ were on no treatment, namely those with PPMS and SPMS. The percentage of patients receiving immunoprophylaxis varies among studies. In an Australian cohort, 65\% of RRMS patients received DMTs and $81.6 \%$ had used at least one DMT at some point, while $18.4 \%$ were never treated..$^{36}$ In a German registry in $2008,71 \%$ received immunomodulatory treatment and $20 \%$ did not receive treatment. ${ }^{15}$ In our series, the number of patients on fingolimod tripled in the last assessment compared to the initial evaluation. This was likely related to the recent governmental sponsorship of this treatment. On the other hand, treatment options like glatiramer acetate, natalizumab, and the new oral treatments were infrequently used, due to unavailability or high cost for the majority of patients who did not have insurance coverage.

The formulation of the results of this study, being pooled from different centers, required a meticulous and standardized patient record-review procedure, which adds strength to this work. Moreover, the analyses of different variables that may affect the disease course can help with early identification of high-risk patients amenable to disease progression. On the other hand, some challenges that faced both patients and physicians were revealed, such as the unavailability of some DMTs. One limitation was the use of different imaging protocols with different scanners among patients, which made radiographic comparison a bit difficult, and also the fact that spinal cord MRIs were not conducted for all patients, resulting in some shortage of data. Another limitation was related to different lab techniques used in CSF analyses and Aqp4-antibody assessment. Generally, the need for nationwide unification of patients' data urged the MS researchers in Egypt to adopt a standardized electronic registry among all universities and major medical institutes, which has been recently established, to allow for more multicenter studies with more homogeneous data.

\section{Conclusion}

The results of this registry study confirm that the demographics and clinical and paraclinical data of Egyptian MS patients are comparable with other registries in the MENA region and in Europe. However, a slightly lower mean age at onset and lower incidence of family history could be attributed to case ascertainment, differences in study design, and some cultural norms.

\section{Disclosure}

The authors report no conflicts of interest in this work.

\section{References}

1. Ascherio A. Environmental factors in multiple sclerosis. Expert Rev Neurother. 2013;13(12 Suppl):3-9.

2. Jennum P, Wanscher B, Frederiksen J, Kjellberg J. The socioeconomic consequences of multiple sclerosis: a controlled national study. Eur Neuropsychopharmacol. 2012;22(1):36-43.

3. Grossman I, Miller A. Multiple sclerosis pharmacogenetics: personalized approach towards tailored therapeutics. EPMA J. 2010;1(2):317-327.

4. Browne P, Chandraratna D, Angood C, et al. Atlas of Multiple Sclerosis 2013: a growing global problem with widespread inequity. Neurology. 2014;83(11):1022-1024.

5. Deleu D, Mir D, Al Tabouki A, et al. Prevalence, demographics and clinical characteristics of multiple sclerosis in Qatar. Mult Scler. 2013; 19(6):816-819.

6. Alroughani R, Ahmed SF, Behbahani R, et al. Increasing prevalence and incidence rates of multiple sclerosis in Kuwait. Mult Scler. 2014; 20(5):543-547.

7. Hashem S, El-Tamawy MS, Hamdy S, Elmasry T. Epidemiology of multiple sclerosis in Egypt. Egypt J Neurol Psychiatry Neurosurg. 2010;47(4):625-632.

8. El-Tallawy HN, Farghaly WM, Badry R, et al. Prevalence of multiple sclerosis in Al Quseir city, Red Sea Governorate, Egypt. Neuropsychiatr Dis Treat. 2016;12:155-158.

9. Polman CH, Reingold SC, Banwell B, et al. Diagnostic criteria for multiple sclerosis: 2010 revisions to the McDonald criteria. Ann Neurol. 2011;69(2):292-302.

10. Lublin FD, Reingold SC. Defining the clinical course of multiple sclerosis: results of an international survey. Neurology. 1996;46(4): 907-911.

11. Wingerchuk DM, Banwell B, Bennett JL, et al. International consensus diagnostic criteria for neuromyelitis optica spectrum disorders. Neurology. 2015;85(2):177-189.

12. Okuda DT, Mowry EM, Beheshtian A, et al. Incidental MRI anomalies suggestive of multiple sclerosis: the radiologically isolated syndrome. Neurology. 2009;72(9):800-805.

13. Heydarpour P, Khoshkish S, Abtahi S, Moradi-Lakeh M, Sahraian MA. Multiple sclerosis epidemiology in Middle East and North Africa: a systematic review and meta-analysis. Neuroepidemiology. 2015; 44(4):232-244.

14. Kister I, Bacon TE, Chamot E, et al. Natural history of multiple sclerosis symptoms. Int J MS Care. 2013;15(3):146-158.

15. Flachenecker P, Stuke K, Elias W, et al. Multiple sclerosis registry in Germany: results of the extension phase 2005/2006. Dtsch Arztebl Int. 2008;105(7):113-119.

16. Chen H, Liu SM, Zhang XX, et al. Clinical features of patients with multiple sclerosis and neuromyelitis optica spectrum disorders. Chin Med J (Engl). 2016;129(17):2079-2084.

17. Pittock SJ, Lucchinetti CF. Neuromyelitis optica and the evolving spectrum of autoimmune aquaporin-4 channelopathies: a decade later. Ann N Y Acad Sci. 2016;1366(1):20-39.

18. Disanto G, Ramagopalan SV. On the sex ratio of multiple sclerosis. Mult Scler. 2013;19(1):3-4.

19. Hakim EA, Bakheit AM, Bryant TN, et al. The social impact of multiple sclerosis: a study of 305 patients and their relatives. Disabil Rehabil. 2000;22(6):288-293. 
20. Nielsen NM, Westergaard T, Frisch M, et al. Type 1 diabetes and multiple sclerosis: a Danish population-based cohort study. Arch Neurol. 2006;63(7):1001-1004.

21. Alemany-Rodríguez MJ, Aladro Y, Amela-Peris R, et al. [Autoimmune diseases and multiple sclerosis]. Rev Neurol. 2005;40(10):594-597. Spanish.

22. Fernández O, Fernández V, Arbizu T, et al. Characteristics of multiple sclerosis at onset and delay of diagnosis and treatment in Spain (the Novo study). J Neurol. 2010;257(9):1500-1507.

23. El-Salem K, Al-Shimmery E, Horany K, Al-Refai A, Al-Hayk K, Khader Y. Multiple sclerosis in Jordan: a clinical and epidemiological study. J Neurol. 2006;253(9):1210-1216.

24. Yamout B, Barada W, Tohme RA, Mehio-Sibai A, Khalifeh R, El-Hajj T. Clinical characteristics of multiple sclerosis in Lebanon. J Neurol Sci. 2008;270(1-2):88-93.

25. Al-Araji A, Mohammed AI. Multiple sclerosis in Iraq: does it have the same features encountered in Western countries? J Neurol Sci. 2005; 234(1-2):67-71

26. Kolber P, Montag S, Fleischer V, et al. Identification of cortical lesions using DIR and FLAIR in early stages of multiple sclerosis. J Neurol. 2015;262(6):1473-1482.

27. Sponsler JL, Kendrick-Adey AC. Seizures as a manifestation of multiple sclerosis. Epileptic Disord. 2011;12(4):401-410.

28. Inshasi J, Thakre M. Prevalence of multiple sclerosis in Dubai, United Arab Emirates. Int J Neurosci. 2011;121(7):393-398.
29. Mygland A, Trydal T, Vinje BU, Vedeler C. Isoelectric focusing is superior to immunofixation electrophoresis in diagnosing CNS inflammation. Acta Neurol Scand. 2007;115(2):122-125.

30. Giovannoni G. Cerebrospinal fluid analysis. Handb Clin Neurol. 2014; 122:681-702.

31. Minneboo A, Barkhof F, Polman CH, Uitdehaag BM, Knol DL, Castelijns JA. Infra-tentorial lesions predict long-term disability in patients with initial findings suggestive of multiple sclerosis. Arch Neurol. 2004;61(2):217-221.

32. Popescu V, Agosta F, Hulst HE, et al. Brain atrophy and lesion load predict long term disability in multiple sclerosis. J Neurol Neurosurg Psychiatry. 2013;84(10):1082-1089.

33. Bsteh G, Ehling R, Lutterotti A, et al. Long term clinical prognostic factors in relapsing-remitting multiple sclerosis: insights from a 10-year observational study. PLoS One. 2016;11(7):e0158978.

34. Jarius S, Franciotta D, Paul F, et al. Testing for antibodies to human aquaporin-4 by ELISA: sensitivity, specificity, and direct comparison with immunohistochemistry. J Neurol Sci. 2012;320(1-2):32-33.

35. Jarius S, Wildemann B. AQP4 antibodies in neuromyelitis optica: diagnostic and pathogenetic relevance. Nat Rev Neurol. 2010;6(7): 383-392.

36. Jokubaitis VG, Spelman T, Lechner-Scott J, et al. The Australian Multiple Sclerosis (MS) immunotherapy study: a prospective, multicentre study of drug utilisation using the MSBase platform. PLoS One. 2013;8(3):e59694.
Neuropsychiatric Disease and Treatment

\section{Publish your work in this journal}

Neuropsychiatric Disease and Treatment is an international, peerreviewed journal of clinical therapeutics and pharmacology focusing on concise rapid reporting of clinical or pre-clinical studies on a range of neuropsychiatric and neurological disorders. This journal is indexed on PubMed Central, the 'PsycINFO' database and CAS,

\section{Dovepress}

and is the official journal of The International Neuropsychiatric Association (INA). The manuscript management system is completely online and includes a very quick and fair peer-review system, which is all easy to use. Visit http://www.dovepress.com/testimonials.php to read real quotes from published authors. 